\title{
Gesundheitliche Effekte der Feinstaubbelastung - aktueller wissenschaftlicher Kenntnisstand
}

\author{
Health Effects of Particulate Matter Exposure: Current Scientific Knowledge
}

Autoren

Institute
D. A. Groneberg' ${ }^{1}$ P. Morfeld², T. Kraus ${ }^{3}$, D. Köhler ${ }^{4}$, N. Krug ${ }^{5}$, H. Magnussen ${ }^{6}$, D. Nowak ${ }^{7}$, K. F. Rabe ${ }^{8}$, G. SchultzeWerninghaus ${ }^{9}$, H. Schulz ${ }^{10}$, H. Teschler ${ }^{11}$, C. Vogelmeier ${ }^{12}$, U. Wagner ${ }^{13}$, T. Welte ${ }^{14}$, T. Voshaar ${ }^{15}$, C. Witt ${ }^{16}$

Die Institutsangaben sind am Ende des Beitrags gelistet. eingereicht 6.5.2009

akzeptiert 12.5 .2009

\section{Bibliografie}

DOI 10.1055/s-0029-1214788

Pneumologie 2009; 63:

363-368 ๔ Georg Thieme

Verlag KG Stuttgart · New York ISSN 0934-8387

\section{Korrespondenzadresse \\ Dr. med. Univ.-Prof. \\ David Groneberg}

Institut für Arbeitsmedizin

Charité - Universitätsmedizin

Berlin

Freie Universität Berlin und Humboldt-Universität zu Berlin Thielallee 69-73

14195 Berlin

arbeitsmedizin@charite.de

\section{Zusammenfassung \\ $\nabla$}

Einleitung: Die Luftqualität ist nicht nur für die Homöostase des Atemtrakts, sondern für die des gesamten Organismus von entscheidender Bedeutung. In den letzten Jahren wurden Überschreitungen der durch die Europäische Union festgesetzten Höchstgrenzen von Feinstaubkonzentrationen festgestellt.

Methodik: Die vorliegende Arbeit ist eine selektive Literaturübersicht zur Epidemiologie und zu pathophysiologischen Effekten der Feinstaubbelastung.

Ergebnisse: Eine Vielzahl epidemiologischer Studien weist auf eine Assoziation von chronischer Feinstaubbelastung und Gesamtmortalität hin. Am deutlichsten sind die Auswirkungen bei Menschen mit Disposition für kardiovaskuläre und respiratorische Erkrankungen. Allerdings müssen die Effekte von Luftschadstoffen kritisch evaluiert und die Plausibilität einzelner Grenzwerte überprüft werden.

Diskussion: Die negativen Einflüsse der chronischen Feinstaubbelastung sind mittlerweile durch eine große Anzahl epidemiologischer und experimenteller Studien belegt. Aus Sicht der Primärprävention kommt der Luftreinhaltung eine wesentliche Bedeutung zu. Dies betrifft sowohl den Bereich der Außenluft mit Feinstaub und anderen Schadstoffen als auch den Bereich der Innenraumluft mit Tabakrauch.

\section{Abstract \\ $\nabla$}

Introduction: Air quality is not only important for respiratory health but it also influences the homeostasis of the whole human organism. In the past years numerous violations of European Union particulate matter thresholds have been recorded.

Methods: The present study is a selective literature analysis encompassing the epidemiology and pathophysiological effects of particulate matter.

Results: Epidemiological studies point to an association between chronic particulate matter exposure and mortality. The most prominent effects on the human body are present in subjects with cardiovascular or respiratory conditions. However, the effects of air pollutants need to be examined critically and the plausibility of thresholds should be evaluated in detail.

Discussion: The negative influences of chronic particulate matter exposure have been proven by a multitude of epidemiological and experimental studies. From the viewpoint of primary prevention, air quality plays a crucial role. This encompasses both the outdoor compartment with particulate matter and other pollutants and the indoor compartment with tobacco smoke.

\section{Einleitung}

\section{$\nabla$}

In den letzten Jahren haben innerstädtische Messstationen Überschreitungen der durch die Europäische Union festgesetzten Höchstgrenze ( $\bullet$ Tab. 1) von Feinstaubmassenkonzentrationen der Kategorie PM10 ( $\bullet$ Tab. 2 ) ergeben.

Die folgenden Diskussionen in Medizin, Politik und Gesellschaft über Luftverschmutzung mün- 
Tab. 1 Neue EU-Grenzwerte.

$\begin{array}{lll}\text { Mittelungszeit } & \text { Grenzwert } & \text { Einzuhalten bis... } \\ \text { 24 Stunden } & \begin{array}{l}50 \mu \mathrm{\mu g} / \mathrm{m}^{3} \mathrm{PM} 10 \\ 35 \mathrm{Überschreitungen}\end{array} & 01.01 .2005 \\ & 40 \mu \mathrm{g} / \mathrm{m}^{3} \mathrm{PM} 10 & 01.01 .2005 \\ \text { 1 Jahr } & 200 \mu \mathrm{g} / \mathrm{m}^{3} \mathrm{NO}_{2} & 01.01 .2010 \\ 1 \mathrm{Stunde} & 18 \mathrm{Überschreitungen} & \\ & 1 \mathrm{Jahr} 40 \mu \mathrm{\mu g} / \mathrm{m}^{3} \mathrm{NO}_{2} & 01.01 .2010\end{array}$

Tab. 2 Feinstaubpartikel-Definitionen und Einteilungen: „Umweltmedizin“.

\begin{tabular}{|c|c|}
\hline Schlagwort & Beschreibung \\
\hline Feinstaub & $\begin{array}{l}\text { Alle festen und/oder flüssigen Teilchen, die } \\
\text { in der Umgebungsluft suspendiert sind, } \\
\text { werden als Feinstaub bzw. Schwebstaub } \\
\text { bezeichnet. Die internationale Bezeichnung } \\
\text { lautet Particulate Matter (PM), wobei die } \\
\text { physikalischen und biologischen Eigen- } \\
\text { schaften des Feinstaubs von der Größe der } \\
\text { Partikel und ihrer chemischen Zusammen- } \\
\text { setzung bestimmt werden. }\end{array}$ \\
\hline Partikelklassifikation & $\begin{array}{l}\text { Ein wichtiges Einteilungskriterium ist der } \\
\text { Partikeldurchmesser, der von wenigen } \\
\text { Nanometern bis zu ca. } 100 \text { Mikrometern } \\
\text { reicht. Partikel ab einem Durchmes- } \\
\text { ser } \geq 0,1 \mu \mathrm{m} \text { werden durch den aerodynami- } \\
\text { schen Durchmesser }\left(d_{\mathrm{ae}}\right) \text { beschrieben. }\end{array}$ \\
\hline PM10-Definition & $\begin{array}{l}\text { Feinstaub der Kategorie PM10 umfasst alle } \\
\text { Partikel, die einen größenselektierenden } \\
\text { Lufteinlass passieren, der für Referenzparti- } \\
\text { kel mit einem aerodynamischen Durch- } \\
\text { messer von } 10 \mu \mathrm{m} \text { ( } 2,5 \mu \mathrm{m} \text { für PM2.5) eine } \\
\text { Abscheidewirksamkeit von } 50 \% \text { besitzt } \\
\text { (Definition aus EU-Richtlinien). }\end{array}$ \\
\hline PM2.5-Definition & $\begin{array}{l}\text { Feinstaub der Kategorie PM2.5 umfasst alle } \\
\text { Partikel, die einen größenselektierenden } \\
\text { Lufteinlass passieren, der für Referenzparti- } \\
\text { kel mit einem aerodynamischen Durch- } \\
\text { messer von } 2,5 \mu \text { m eine Abscheidewirksam- } \\
\text { keit von } 50 \% \text { besitzt (Definition aus EU- } \\
\text { Richtlinien). }\end{array}$ \\
\hline Probentnahmesysteme & $\begin{array}{l}\text { Entnahmesysteme für die einzelnen PM- } \\
\text { Größen werden durch die europäische Norm } \\
\text { EN } 12341 \text { beschrieben. }\end{array}$ \\
\hline
\end{tabular}

Die Arbeit kann sich aufgrund der Textbegrenzung nicht umfassend mit den wichtigen Themen der Feinstaubexposition durch Passivrauch und der Exposition gegenüber Innenraumnoxen befassen.

Grundsätzlich muss zur Vermeidung von Missverständnissen festgehalten werden, dass weder die Kategorie PM10 noch PM2.5 dem in Deutschland üblichen Feinstaubbegriff, d.h. dem alveolengängigen Staub („A-Staub“, @ Tab. 3), entsprechen.

Dieser wurde von der Senatskommission der Deutschen Forschungsgemeinschaft zur Prüfung gesundheitsschädlicher Arbeitsstoffe („MAK-Kommission“) definiert und ist in der Arbeitsmedizin allgemein gültig.

Die Massenbelastung durch Partikel ist, so wissenschaftlich eindeutig belegt, in den letzten fünfzig Jahren in Deutschland gesunken. Nicht nachweisbar ist, ob die Belastung in bestimmten Regionen größer geworden ist [1].
Tab. 3 Staubpartikel-Definitionen und Einteilungen: „Arbeitsmedizin“.

\begin{tabular}{ll}
$\begin{array}{l}\text { Schlagwort } \\
\text { Einatembare Fraktion: }\end{array}$ & $\begin{array}{l}\text { Beschreibung } \\
\text { Der Masseanteil aller Schwebstoffe, } \\
\text { der durch Nase und Mund eingeatmet wird. }\end{array}$ \\
\hline $\begin{array}{l}\text { Extrathorakale } \\
\text { Staubfraktion: }\end{array}$ & $\begin{array}{l}\text { Masseanteil der eingeatmeten Partikel, der } \\
\text { bis zum Kehlkopf, aber nicht über diesen } \\
\text { hinaus in die Atemwege gelangen kann. }\end{array}$ \\
\hline $\begin{array}{l}\text { Tracheobronchiale } \\
\text { Staubfraktion: }\end{array}$ & $\begin{array}{l}\text { Masseanteil der eingeatmeten Partikel, } \\
\text { der über den Kehlkopf hinaus, aber nicht } \\
\text { bis in den nichtzilierten Bereich der Lunge } \\
\text { (Alveolen) gelangen kann. }\end{array}$ \\
\hline $\begin{array}{l}\text { Alveolengängige } \\
\text { Staubfraktion (A-Staub): }\end{array}$ & $\begin{array}{l}\text { Masseanteil der eingeatmeten Partikel, } \\
\text { der bis in den nichtzilierten Bereich der } \\
\text { Lunge gelangen kann. }\end{array}$ \\
\hline Anzahlkonzentration & Partikelanzahl pro Luftvolumen \\
\hline Massenkonzentration & Partikelmasse pro Luftvolumen
\end{tabular}

Zur Bewertung der Luftqualität wird weltweit die bodennahe Atmosphäre auf die Massenbelastung durch Partikel untersucht. Dabei wird ein in den letzten Jahren vollzogener Paradigmenwechsel in der Aerosoltoxikologie oft außer Acht gelassen [2,3]. Viele der neuen Studien zeigen, dass es nicht wie früher angenommen die Partikelmasse ist, die in Korrelation zu potenziellen Gesundheitsschäden steht, sondern die Anzahlkonzentration der Partikel sowie die Partikeloberfläche und die Zusammensetzung. Besonders die ultrafeinen Partikel mit wenig Masse stellen durch ihre hohe Anzahl und große Oberfläche eine Gefahr für die Lunge als Eintrittsorgan, für das kardiovaskuläre System und eventuell sogar für das zentrale Nervensystem dar [4]. Dieselrußpartikel, die zum größten Teil aus Feinstaubpartikeln bestehen, werden als ein wichtiger Faktor für die Überschreitung der zugelassenen Höchstgrenze der Feinstaubkonzentration diskutiert. Eine Maßnahme zur effektiven Reduktion dieses Schadstoffes ist der Dieselrußfilter, durch den eine Verminderung dieser Emission um rund $20 \%$ erreicht werden kann [5]. Trotzdem kann dadurch eine deutliche Reduktion des insgesamt in Deutschland gemessenen PM10, und damit eine Einhaltung des Grenzwertes für PM10, durch diese Maßnahme nicht erwartet werden. Dennoch ist die Reduktion der Dieselrußpartikel unter Berücksichtigung der neuen aerosoltoxikologischen Erkenntnisse ein wichtiges Vorhaben.

Bisher ungeklärt ist die Höhe und die Bedeutung der Belastung in Innenräumen im Vergleich zur Außenluft. Hier ist zu bedenken, dass sich die Menschen in modernen Industriegesellschaften nur noch etwa 10\% des Tages im Freien aufhalten [6]. Etwa 70\% der Partikelmenge in der Außenluft werden laut Untersuchungen auch in der Raumluft gefunden [7]. Bei der Innenraumluft kommen allerdings noch erhebliche Belastungen durch so genannte Indoor-Partikel dazu, wie besonders vom Tabakrauch, aber auch von Kerzen und offenem Feuer oder vom Kochen. Die Belastung durch den Tabakrauch untersucht eine Studie, die in Nichtraucher-Restaurants eine Partikelmasse von $57 \mu \mathrm{g} / \mathrm{m}^{3}$ gefunden hat und in Raucher-Restaurants einen Durchschnitt von $190 \mu \mathrm{g} / \mathrm{m}^{3}$ mit Spitzenwerten bis zu $400 \mu \mathrm{g} / \mathrm{m}^{3}$ [8]. Bei einer Messung von PM2.5 fanden Travers u. Mitarb. [9] in New Yorker Restaurants sogar Durchschnittswerte von $412 \mu \mathrm{g} / \mathrm{m}^{3}$ (Spitzenwert $1375 \mu \mathrm{g} /$ $\mathrm{m}^{3}$ ), die nach dem Rauchverbot in öffentlichen Gebäuden in den gleichen Restaurants auf einen durchschnittlichen Wert von $27 \mu \mathrm{g} / \mathrm{m}^{3}$ gesunken waren. 
Epidemiologische Aspekte der Feinstaubwirkungen

Die WHO hat im Jahr 2005 die in $\bullet$ Abb. 1 skizzierten relativen Risiken für Mortalität im Zusammenhang mit einer Zunahme der Schadstoffkonzentration um $10 \mu \mathrm{g} / \mathrm{m}^{3}$ publiziert.

Es wird mittlerweile häufig diskutiert, dass diese Zahlen weitaus niedriger sind als Werte für Hypertonie oder Tabakrauchen. Einen signifikanten statistischen Zusammenhang zwischen der Zunahme der Gesamtmortalität, der Mortalität von Krebs und kardiopulmonalen Erkrankungen einerseits und der zunehmenden Partikelbelastung in der Außenluft andererseits konnten drei von vier großen amerikanischen Kohortenstudien, nämlich die Harvard Six City Study [10], die American Cancer Society Study [11] und die Adventist Health Study [12,6], nachweisen. Krewski [13] reanalysierte die Studien von Dockery [23] und Pope [14] und bestätigte die initialen Befunde. Auf der anderen Seite führte eine durch Pope [15] durchgeführte Reanalyse zu widersprüchlichen Ergebnissen, sodass noch keine abschließende und umfassende Bewertung möglich ist [15]. Das relative Risiko scheint dabei auch vom Ausbildungsgrad abhängig zu sein. Entweder sind verwendete Variablen zum sozioökonomischen Status ungenügend berücksichtigt worden oder es könnte sich hier ein Schwellenwert andeuten [15]. Zur gesundheitlichen Bedeutung von Feinstaub wurden auch in Europa zwei longitudinale Studien publiziert [16,17]. Hoek [17] konnte dabei eine Zunahme der kardiopulmonalen Mortalität in Korrelation zur Wohnnähe an Hauptverkehrsstraßen aufzeigen. Ein Zusammenhang der partikulären Luftverschmutzung mit der Kindersterblichkeit konnte gleich von mehreren Studien gezeigt werden [18-22]. Von großem Interesse ist dabei auch die Studie von Clancy [e23], die eine Intervention untersucht. Hier werden methodische Probleme deutlich: Das Verbot des Kohleverkaufs in Dublin führte ab dem 1. September 1990 zu einer erheblichen Reduktion der Black-Smoke-Konzentration (korreliert weitgehend mit PM10, vgl. Goodman et al. 2004 [24], und es wurde eine Senkung von nicht-traumatischen Todesfällen von 5,7\% (95\% CI 4-7, $\mathrm{p}<0,0001)$, respiratorischen Todesfällen von 15,5\% (12-19, $\mathrm{p}<0,0001)$ und kardiovaskulären Todesfällen von 10,3\% (8-13, $\mathrm{p}<0,0001$ ) berichtet. Allerdings wurden die Ergebnisse dieser Studie in den Folgejahren reanalysiert und die Autoren kamen selbst zu dem Schluss, dass wichtige Störfaktoren wie beispielsweise Wettereinflüsse anscheinend nicht ausreichend berücksichtigt wurden [24]. In einer aktuellen Arbeit von Wittmaack [25] wird zudem festgestellt, dass die Ergebnisse der Studie von Clancy [16] auch deswegen limitiert seien, da die dem Staub zugeschriebenen Effekte wesentlich durch nicht hinreichend berücksichtigten Infektionswellen mitverursacht waren. Die Diskussion um die tatsächlichen Auswirkungen hat mittlerweile die breite Öffentlichkeit erreicht und die Effekte der Immissionsbegrenzungsmaßnahmen wurden diesbezüglich mittlerweile kritisch diskutiert [26]. Wird in dieser Diskussion auf genau eine Störgröße (hier: Grippewelle) abgehoben, die nach Wittmaack 2007 nicht genügend in den Analysen betrachtet wurde, so leitet dieses Beispiel zur „multiple bias“-Problematik über, die z.B. aufgrund nicht oder ungenügend vermessener Confounder entstehen kann - eine bislang zu gering beachtete Schwierigkeit bei Studien mit geringen Wirkungen (kleines relatives Risiko), aber hoher Präzision (geringe Varianz des relativen Risikos wegen vieler Studienteilnehmer). Die Möglichkeit der ungünstigen Überlagerung mehrerer in ihrer Größe durchaus plausibler Verzerrungen kann vordergründig signifikante, positive Befunde bewirken. Als Beispiel sei hierzu die Untersuchung von Greenland im Jahr 2005 zur Wirkung elektromagnetischer Felder durch Hochspan-

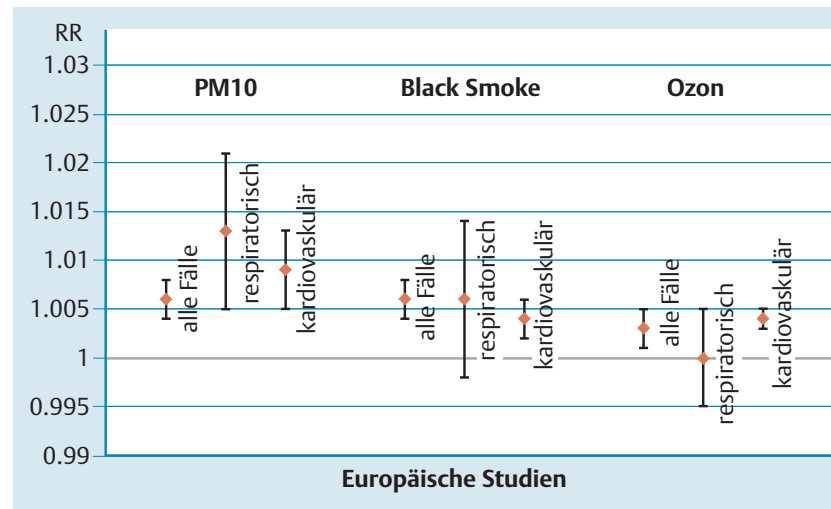

Abb. 1 Relatives Risiko (RR) für Mortalität bezogen auf Zunahme der Luftschadstoffkonzentration um $10 \mathrm{\mu g} / \mathrm{m}^{3}$ (95\% Konfidenzintervall). Modifiziert nach [63].

nungsleitungen auf das Risiko der Entwicklung kindlicher Leukämien angeführt [27]. Es konnte dabei gezeigt werden, dass die vordergründig homogenen Befunde der Risikoerhöhung plausibel durch die Überlagerung unkontrollierter Störfaktoren erklärbar sind. Eine solche Problematik wird auch für den Bereich der Umweltstäube als möglich gesehen - entsprechend wird von vielen Arbeitsgruppen eine geeignete bias-Modellierung gefordert [28-32].

Einen deutlichen Zusammenhang zwischen Erkrankungen des pulmonalen Systems und der Schadstoffbelastung durch Aerosole zeigen jedoch eine Reihe amerikanischer, europäischer und deutscher Querschnittsstudien. So zeigen die Arbeiten von Braun-Fahrländer [33], Dockery [23], Heinrich [34,35], Krämer [36], McConnell [37] und Raizenne [38] eine Korrelation zwischen PM10-Exposition und pulmonalen Pathologien wie Bronchitis, Husten oder pfeifenden Atemgeräuschen. Eine Assoziation zwischen partikulärer Luftbelastung und eingeschränkter Lungenfunktion zeigen die Veröffentlichungen von Ackermann-Liebrich [39] und Zemp [40]. Bei Kindern ist unter erhöhten Staubbelastungen der Luft das Lungenwachstum reduziert, wie Avol [41], Jedrychowski [42] sowie Gauderman [43,44] nachweisen konnten. Die Interpretation aller epidemiologischen Studien lässt insgesamt den Schluss zu, dass die Langzeitexposition gegenüber partikulären Luftschadstoffen speziell für suszeptible Personen (Kinder, Kranke, Senioren) ein Gesundheitsrisiko darstellen kann. Eine Zunahme respiratorischer Beschwerden wie Husten, Auswurf und Kurzatmigkeit, eine leichte Einschränkung der Lungenfunktion sowie ein vermehrter Bedarf an Medikamenten lässt sich bei exponierten Asthmatikern nachweisen. Dabei sind die Symptome häufiger bei Erwachsenen zu finden als bei Kindern. Auch bei der Auswertung von Feldstudien ergeben sich bei Asthmatikern Hinweise auf eine Verschlechterung der Lungenfunktion in Abhängigkeit von der Feinstaubbelastung [45]. Außerdem konnten bei den Betroffenen eine verminderte Variabilität der Herzfrequenz und ein vermehrtes Auslösen von implantierten Kardiovertern sowie eine Erhöhung des C-reaktiven Proteins und der Viskosität des Plasmas festgestellt werden $[46,47]$. Betroffen sind daneben auch Patienten mit Diabetes mellitus und kardiovaskulären Vorerkrankungen [48].

Eine Abnahme der mittleren geschätzten Lebenserwartung von 9-12 Monaten in Mitteleuropa bzw. die Zahl von 65000 errechneten vorzeitigen Todesfällen infolge von umweltbedingten Luftschadstoffen hat vor kurzem das Clean Air for Europe (CAFE)Programm veröffentlicht [49]. Die im CAFE-Dokument durchge- 
führten Analysen der „Zusatztoten“ durch Staubbelastungen gehen davon aus, dass die belasteten Menschen „mehrfach“ sterben können, wodurch schließlich die Zahl der 65000 Zusatztoten entsteht. Diesbezüglich muss methodenkritisch angemerkt werden, dass aus kausallogischen Gründen die Erhöhung einer Staubexposition nicht zu zusätzlichen Todesfällen führen kann, da jeder Mensch „nur einmal sterben“ kann [50,51]. Dieser Aspekt wurde ebenfalls bereits in der Cost-Benefit-Analysis zum CAFE-Programm ausgeführt [52]. Es muss auch darauf verwiesen werden, dass es unterschiedliche Ergebnisse erheblichen Ausmaßes bei der Risikoabschätzung gibt. Dabei hat z. B. Roller die seit Januar 2005 geltenden EU-Feinstaubgrenzwerte mit den Zielwerten des Länderausschusses für Immissionsschutz (LAI) von 1992 und aktuellen Messdaten verglichen und mögliche Krebsrisiken analysiert [53]. Es wurde unter Berücksichtigung relevanter Kanzerogene aus der Summe der Risiken für das Szenario einer fortwährenden Exposition einer Population von 80 Mio. Individuen gegenüber $3 \mu \mathrm{g} / \mathrm{m}^{3}$ PM2.5 eine Rate von unter 200 expositionsbedingten Lungenkrebstodesfällen pro Jahr geschätzt. Diese Schätzung war um den Faktor 10 niedriger als die Risikoabschätzung von Wichmann aus dem Jahr 2004 [54]. Diese ging allerdings von anderen Grundlagen aus. Es wurde dabei die Wirkung von $3 \mu \mathrm{g} / \mathrm{m}^{3}$ PM2.5 mit der von Dieselruß von $3 \mu \mathrm{g} / \mathrm{m}^{3}$ gleichgesetzt und keine Wirkungsschwelle angenommen. Im Unterschied dazu diskutierte Roller, dass am Arbeitsplatz gemeinhin von einer Wirkungsschwelle für nicht spezifisch toxische Stäube bei Exposition in Höhe des Allgemeinen Staubgrenzwerts ausgegangen werden muss. Die Differenzen zwischen den einzelnen Studien bedürfen weiterer Klärung.

Aus epidemiologischer Sicht besteht ein Zusammenhang zwischen umweltbedingter Partikelbelastung und biologischen Kurzzeiteffekten. Von den erfassten Daten ist derzeit allerdings eine biologisch wirksame Schwellenkonzentration von Atemluftpartikeln noch nicht ableitbar [55]. Aufgrund der sehr komplexen Struktur von Zeitreihen-Studien können diese im Allgemeinen ebenfalls nicht als klarer Kausalbeweis eines Zusammenhangs zwischen Umweltstaubexpositionen und der Tagesmortalität angesehen werden.

Eine Schwellenkonzentration für Entzündungsreaktionen im Atemtrakt nach Instillation ultrafeiner Kohlenstoffpartikel konnte aber im Tierexperiment ermittelt werden [56]. Sie wird als die Oberfläche der Partikel definiert (etwa $20 \mathrm{~cm}^{2}$ ), die in Lungen von Mäusen deponiert wurde und gerade noch keine Entzündungsreaktion hervorgerufen hat. $20 \mathrm{~cm}^{2}$ entsprechen etwa $2 \%$ der Lungenoberfläche einer Maus. Keine entzündlichen Reaktionen werden dagegen bei einer Applikation von Partikeln mit einer kleineren Oberfläche beobachtet.

\section{Mögliche pathophysiologische Effekte} der Staubexposition

Aufgrund von epidemiologischen Metaanalysen wurde geschätzt, dass ein akuter Anstieg der Feinstaubbelastung (PM10) um $10 \mu \mathrm{g} / \mathrm{m}^{3}$ die Mortalität im Mittel um 0,6\% (0,4\%-0,8\%) erhöhen kann. Dabei nimmt die Mortalität aufgrund von Lungenerkrankungen im Mittel um 1,3\% (0,5\%-2,0\%) die kardiovaskuläre Mortalität um 0,9\% (0,5\%-1,3\%) zu [57]. Zwar ist der prozentuale Anstieg der Mortalität durch Herz-Kreislauf-Erkrankungen geringer als der durch Lungenerkrankungen, er ist aber für die Gesamtbevölkerung von größerer Bedeutung, da die kardiovaskuläre die pulmonale Morbidität und Mortalität deutlich übersteigt. Als Todesursachen wurden Rhythmusstörungen, Herzinfarkte und Herzversagen festgestellt.
Die in epidemiologischen Studien mit einer verstärkten Feinstaubexposition einhergehenden Veränderungen im Herz-Kreislauf-System sind vielfältig und sprechen für komplexe systemische Reaktionen. Unter anderem wurden ein Herzfrequenzanstieg bei eingeschränkter Herzfrequenzvariabilität sowie eine arterielle Vasokonstriktion mit Anstieg des Blutdrucks beschrieben $[19,18,32]$. Im Blut wurden eine erhöhte Plasmaviskosität, ein Anstieg von Fibrinogen und C-reaktivem Protein [31] sowie die Aktivierung von Thrombozyten [47] beobachtet.

Die den kardiovaskulären Veränderungen zugrunde liegenden pathophysiologischen Mechanismen sind bisher nur ansatzweise entschlüsselt worden, allerdings gibt es weltweit akzeptierte Arbeitshypothesen. Postuliert werden unter anderem:

a) eine Störung des vegetativen Gleichgewichtes durch entzündliche oder Stressreaktionen in der Lunge und/oder durch rezeptorvermittelte pulmonale Reflexe

b) eine pulmonale oder systemische pro-inflammatorische Reaktionslage mit vermehrtem oxidativen Stress, erhöhter Gerinnungsneigung, Akutphase-Reaktion, endothelialer Dysfunktion und Förderung atherosklerotischer Prozesse

c) eine Beeinflussung der Herzfunktion durch Minderperfusion und/oder veränderter Ionenkanalfunktion der Kardiomyozyten, die zur elektrophysiologischen Instabilität mit verstärktem Auftreten von Arrhythmien führt.

Die der Hypothese einer gestörten autonomen Balance zugrunde liegenden Effekte auf die Parameter Herzfrequenz und Herzfrequenzvariabilität lassen sich unter kontrollierten Bedingungen am gesunden Tier bei Staubkonzentrationen im Spitzenbereich $\left(180 \mu \mathrm{g} / \mathrm{m}^{3}\right.$ über $24 \mathrm{Std}$.) nachweisen [33], wurden aber auch bei älteren, spontan hypertensiven Ratten als Folge einer direkten Exposition mit Straßenverkehrsimmissionen beschrieben [58]. Unter den Studien am Menschen ist eine Untersuchung [59] von Bedeutung, in der Veränderungen von Herzfrequenz und Herzfrequenzvariabilität sowie auch eine Zunahme von supraventrikulären Extrasystolen bei jungen, gesunden Autobahnpolizisten beobachtet wurden. Eine Einschränkung der Herzfrequenzvariabilität gilt als Indikator einer vegetativen Imbalance und als Risikofaktor für das Auftreten von Arrhythmien und plötzlichem Herztod. Während die Auswirkungen einer akut erhöhten Feinstaubbelastung bei Patienten mit entsprechender Disposition nachvollziehbar sind, ist die Bedeutung bei Gesunden mit entsprechenden Kompensationsmöglichkeiten selbst bei chronischer oder wiederholter Belastung noch vollkommen unklar.

Akutphase-Reaktionen (Anstieg von C-reaktivem Protein, Fibrinogen und unter anderem der zirkulierenden Granulozyten) waren sowohl in epidemiologischen als auch in toxikologischen Studien wiederholt nachweisbar und werden durch die Ergebnisse kontrollierter Expositionsstudien am Menschen bestätigt [60]. Die Hypothese eines Feinstaub-induzierten gerinnungs- und thrombosefördernden Status wird insbesondere durch toxikologische Untersuchungen untermauert.

Bei den in Deutschland aktuell vorliegenden Umweltbelastungen muss man davon ausgehen, dass Feinstaub-induzierte Reaktionen - insbesondere beim Gesunden - auf einem geringen, schwer nachweisbaren Niveau ablaufen, die Wirkung aber bei chronischer Belastung akkumuliert. Eindeutig nachweisbare Effekte kommen wahrscheinlich erst nach jahrelanger Feinstaubexposition und mit großer Wahrscheinlichkeit überwiegend bei gleichzeitigem Vorliegen von kardiovaskulären Risikofaktoren oder einer genetischen Disposition zum Tragen. Epidemiologisch kann in diesem Fall im erkrankten Herz-Kreislauf-System eine Häufung von „akuten“ Ereignissen im Sinne von Angina-pecto- 
ris-Anfällen, Arrhythmien und Herzinfarkten als Folge einer erhöhten Feinstaubbelastung demonstriert werden.

Insgesamt unterstreichen die vorliegenden epidemiologischen und toxikologischen Studien, dass eine Korrelation zwischen Feinstaubexposition und kardiorespiratorischen Ereignissen vorhanden ist - jedoch gibt es gerade zur Frage, wer von welchem Feinstaub und in welchem Umfang betroffen ist, noch erheblichen Forschungsbedarf.

\section{Ausblick}

Bislang wird die Luftqualität der Umwelt in Bezug auf die Partikelmasse beurteilt, ohne dabei wichtige arbeitsmedizinisch-toxikologische Aspekte wie die chemische Zusammensetzung oder die Größe und Anzahl der Partikel zu berücksichtigen. Steigt wie diskutiert mit abnehmender Partikelgröße die biologische Relevanz, so wird die toxische Dosis nicht durch die Masse, sprich Größe der Partikel bestimmt, sondern durch deren Oberfläche. Daneben spielen wahrscheinlich auch chemische Eigenschaften der Partikel und die Bindung unterschiedlichster Stoffe (auch Gase) an die Partikeloberfläche eine Rolle. Bezüglich der in der EU seit 2005 festgelegten Feinstaub-Grenzwerte ist festzuhalten, dass trotz voraussichtlicher Senkung des Gesundheitsrisikos nicht vorherzusehen ist, wie groß der erzielbare positive Effekt ausfällt $[61,62]$. Es besteht ein erheblicher Forschungsbedarf, der vor allem Erkenntnisse der Aerosoltoxikologie, Epidemiologie, Pneumologie und Arbeitsmedizin berücksichtigen muss. Auch die epidemiologischen Langzeit-Mortalitätsstudien lassen wichtige Fragen offen wie das multiple-bias-Problem und mögliche Schwellenwerte. Deswegen erscheint ein Schwellenwertund multiple-bias-Modelling der epidemiologischen Daten im Rahmen neuer Studien notwendig.

\section{Feinstaubbelastung}

1. Epidemiologische Studien weisen eindeutig auf eine Assoziation von chronischer Feinstaubbelastung und Gesamtmortalität hin.

2. Am deutlichsten sind die Auswirkungen bei Menschen mit Disposition für kardiovaskuläre und respiratorische Erkrankungen.

3. Es bedarf einer weiteren Analyse und Evaluation der akuten und chronischen Effekte von Luftschadstoffen und einer Überprüfung der Plausibilität einzelner Grenzwerte.

\section{Interessenkonflikt}

$\nabla$

Die Autoren erklären, dass kein Interessenkonflikt im Sinne der Richtlinien des International Committee of Medical Journal Editors besteht.

\section{Institute}

1 Institut für Arbeitsmedizin, Charité - Universitätsmedizin Berlin, Freie Universität Berlin und Humboldt-Universität zu Berlin, Berlin

2 Institut für Epidemiologie und Risikobewertung in der Arbeitswelt (IERA),

Essen und Institut für Arbeits- und Sozialmedizin der Universität zu Köln

3 Institut und Poliklinik für Arbeits- und Sozialmedizin, RWTH Aachen, Aachen

${ }^{4}$ Fachkrankenhaus Kloster Grafschaft, Schmallenberg

5 Abteilung für Immunologie, Allergologie und Atemwegsforschung, Fraunhofer Institut für Toxikologie und Experimentelle Medizin, Hannover

6 Krankenhaus Großhansdorf, Zentrum für Pneumologie und Thoraxchirurgie, Großhansdorf

Institut und Poliklinik für Arbeits- und Umweltmedizin, Ludwig-MaximiliansUniversität, München

8 Klinik für Lungenheilkunde, University Medical Center Leiden, Leiden, Niederlande

9 Medizinische Klinik III, Pneumologie, Allergologie, Schlaf- und Beatmungsmedizin, Berufsgenossenschaftliches Universitätsklinikum Bergmannsheil, Ruhr-Universität Bochum

10 Helmholtz Zentrum München, Institute of Lung Biology and Disease, Neuherberg/München

11 Universitätsklinik für Pneumologie, Ruhrlandklinik, Westdeutsches Lungenzentrum am Universitätsklinikum Essen, Essen

12 Klinik für Innere Medizin, Schwerpunkt Pneumologie, Universitätsklinikum Gießen und Marburg, Marburg

13 Klinik für Pneumologie, Fachklinik Löwenstein, Löwenstein

14 Abteilung Pneumologie, Zentrum Innere Medizin, Medizinische Hochschule Hannover, Hannover

15 Medizinische Klinik III, Krankenhaus Bethanien, Moers

16 Medizinische Klinik m. S. Infektiologie und Pneumologie, Charité - Universitätsmedizin Berlin

\section{Literatur}

1 Kreyling WG, Tuch T, Peters A et al. Diverging long-term trends in ambient urban particle mass and number concentrations associated with emission changes caused by the German unification. Atmos Environ 2003; 37: 3841 - 3848

2 Ferin J, Oberdörster G, Penney DP et al. Increased pulmonary toxicity of ultrafine particles? I. Particle clearance, translocation, morphology. J Aerosol Sci 1990; 21: 381 - 384

3 Oberdörster G, Ferrin J, Finkelstein G et al. Increased pulmonary toxicity of ultrafine particles? II. Lung Lavage Studies. J Aerosol Sci 1990; 21: $384-387$

4 Peters A, Veronesi B, Calderón-Garcidueñas L et al. Translocation and potential neurological effects of fine and ultrafine particles a critical update. Part Fibre Toxicol 2006; 83: 13

5 Wichmann HE. Abschätzung positiver gesundheitlicher Auswirkungen durch den Einsatz von Partikelfiltern bei Dieselfahrzeugen in Deutschland. Gut-BMU-Diesel 7. 6. 2003

6 Seidel HJ. Umweltmedizin. Stuttgart: Thieme, 1996

7 Abbey DE, Nishino N, McDonnell WF. Development of chronic productive cough as associated with long-term ambient inhalable particulate pollutants (PM10) in non-smoking adults. In: Lee J, Phalen R (eds). Proceedings of the second colloquium on particulate air pollution and human health. Salt Lake City: University of Utah, 1996: 401 - 424

8 Brauer M, Mannetje A, Lang B. Continuous assessment of indoor fine particles with a portable nephelometer. In: Lee J, Phalen R (eds). Proceedings of the second colloquium on particulate air pollution and human health. Salt Lake City: University of Utah, 1996: 455-469

9 Travers MJ, Cummings KM, Hyland A et al. Indoor air quality in hospitality venues before and after implementation of a clean indoor air law. MMWR 2004; 53: 1038 - 1041

10 Dockery DW, Pope CA, Xu XP et al. An association between air pollution and mortality in six U.S. cities. N Engl J Med 1993; 329: 1753-1759

11 Pope CA, Burnett RT, Thun MJ et al. Lung cancer, cardiopulmonary mortality, and long-term exposure to fine particulate air pollution. JAMA 2002; 287: 1132 - 1141

12 Abbey DE, Nishino N, McDonnell WF et al. Longterm inhalable particles and other air pollutants related to mortality in nonsmokers. Am J Respir Crit Care Med 1999; 159: 373 - 382

13 Krewski D, Burnett RT, Goldberg MS et al. Reanalysis of the Harvard Six Cities Study and the American Cancer Society Study of particulate air pollution and mortality. Cambridge, MA: Health Effects Institut, 2000

14 Pope CA, Thun MJ, Namboodiri MM et al. Particulate air pollution as a predictor of mortality in a prospective study of US adults. Am J Respir Crit Care Med 1995; 151: 669-674 
15 Pope CA, Burnett RT, Thun MJ et al. Lung cancer, cardiopulmonary mortality, and long-term exposure to fine particulate air pollution. JAMA 2002; 287: 1132 - 1141

16 Clancy L, Goodman P, Sinclair $H$ et al. Effect of air-pollution control on death rates in Dublin, Ireland: An intervention study. Lancet 2002; 360: $1210-1214$

17 Hoek G, Brunekreef B, Goldbohm S et al. Association between mortality and indicators of traffic-related air pollution in the Netherlands: a cohort study. Lancet 2002; 360: 1203 - 1209

18 Bobak M, Leon DA. Air pollution and infant mortality in the Czech republic 1986-88. Lancet 1992; 340: 1010 - 1014

19 Bobak M, Leon DA. Pregnancy outcomes and outdoor air pollution: an ecological study in districts of the Czech Republic 1986-88. Occup Environ Med 1999; 56: 539- 543

20 Dejmek J, Selevan SG, Benes I et al. Fetal growth and maternal exposure to particulate matter during pregnancy. Environ Health Perspect 1999; 107: $475-480$

21 Wodruff TJ, Grillo J, Schoendorf KC. The relationship between selected causes of postneonatal infant mortality and particulate air pollution in the United States. Environ Health Perspect 1997; 105: 608-612

22 Lipfert FW, Zhang J. Wyzga RE. Infant mortality and air pollution: A comprehensive analysis of U.S. data for 1990. J Air Waste Manage Assoc 2000; 50: 1350 - 1366

23 Dockery DW, Cunningham J, Damokosh AI et al. Health effects of acid aerosols on North American children: Respiratory symptoms. Environ Health Perspect 1996; 104: 500-505

24 Goodman PG, Dockery DW, Clancy L. Cause-specific mortality and the extended effects of particulate pollution and temperature exposure. Environ. Health Perspect 2004; 112: 179-185

25 Wittmaack $K$. The big ban on bituminous coal sales revisited: serious epidemics and pronounced trends feign excess mortality previously attributed to heavy black-smoke exposure. Inhal Toxicol 2007; 19: $343-350$

26 Wandtner $R$. Wird das Risiko durch Ruß weit überschätzt? FAZ 4. 4. 2007; Nr. 80: N1

27 Greenland S. Multiple-bias modelling for analysis of observational data. J Royal Stat Soc 2005; 168: 267 - 306

28 Ioannidis JPA. Why most published research findings are false. PLoS Medicine 2005; 2: e124

29 Janes H, Dominici F, Zeger S. Partitioning evidence of association between air pollution and mortality. Epidemiology 2007; 18: 427-428

30 Janes H, Dominici F, Zeger SL. Trends in air pollution and mortality: an approach to the assessment of unmeasured confounding. Epidemiology $2007 ; 18: 416-423$

31 Bukowski J. Do time-series studies contain residual condounding by risk factors for acute health events? Epidemiology 2007; 18: 839

32 Koop G, Tole L. Measuring the health effects of air pollution: To what extent can we really say that people are dying from bad air? J Environ Econom Manag 2004; 47: $30-54$

33 Braun-Fahrländer C, Vuille JC, Sennhauser FH et al. Respiratory health and long-term exposure to air pollutants in Swiss schoolchildren. Am J Respir Crit Care Med 1997; 155: 1042 - 1049

34 Heinrich J, Hölscher B, Wichmann HE. Decline of ambient air pollution and respiratory symptoms in children. Am J Respir Crit Care Med 2000; 161: $1930-1936$

35 Heinrich J, Hölscher B, Frye C et al. Improved air quality in reunified Germany and decreases in respiratory symptoms. Epidemiol 2002; 13: $394-401$

36 Krämer $U$, Behrendt $H$, Dolgner $R$ et al. Airway diseases and allergies in East and West German children during the first 5 years after reunification: time trends and the impact of sulphur dioxide and total suspended particles. Int J Epdemiol 1999; 28: 865 - 873

37 McConnell R, Berhane K, Gilliland $F$ et al. Air pollution and bronchitic symptoms in Southern California children with asthma. Environ Health Perspect 1999; 107: $757-760$

38 Raizenne ME, Damokosh AI, Dockery DW et al. Health effects of acid aerosols on North American children. - Pulmonary function. Environ Health Perspect 1996; 104: 506- 514

39 Ackermann-Liebrich U, Leuenberger P, Schwartz J et al. Lung function and long term exposure to air pollutants in Switzerland. Am J Respir Crit Care Med 1997; 155: 122 - 129

40 Zemp E, Elsässer S, Schindler C et al. Long-term ambient air pollution and respiratory symptoms in adults (SAPALIDA Study). Am J Respir Crit Care Med 1999; 159: 1257-1266
41 Avol EL, Gauderman WJ, Tan SM et al. Respiratory effects of relocating to areas of differing air pollution levels. Am J Respir Crit Care Med 2001; 164: 2067-2072

42 Jedrychowski W, Flak E, Mroz E. The adverse effect of low levels of ambient air pollutants on lung function growth in preadolescent children. Environ Health Perspect 1999; 107: 669-674

43 Gauderman WJ, Vora H, McConnell R et al. Effect of exposure to traffic on lung development from 10 to 18 years of age: a cohort study. Lancet 2007; 369: 571 - 517

44 Gauderman WJ, Avol E, Gilliland F et al. The effect of air pollution on lung development from 10 to 18 years of age. N Engl J Med 2004; 351: $1057-1067$

45 Peters A, Heinrich J, Wichmann HE. Gesundheitliche Wirkungen von Feinstaub - Epidemiologie der Kurzzeiteffekte. Umweltmed Forsch Prax 2002; 7: 101 - 115

46 Peters A, Döring A, Wichmann HE et al. Increased plasma viscosity during the 1985 air pollution episode: a link to mortality? Lancet 1997; 349: $1582-1587$

47 Rückerl R, Phipps RP, Schneider A et al. Ultrafine particles and platelet activation in patients with coronary heart disease - results from a prospective panel study. Part Fibre Toxicol 2007; 4: 1

48 Zanobetti A, Schwartz J, Gold D. Are there sensitive subgroups for the effects of airborne particles? Environ Health Perspect 2000; 108: $841-845$

49 European Commission. CAFÉ CBA: Baseline Analysis 2000 to 2002. Service Contract for Carrying out Cost-Benefit Analysis for Air Quality Related Issues, in particular in the Clean Air for Europe (CAFÉ) Programme. 2005

50 Morfeld $P$. Years of Life Lost due to exposure: Causal concepts and empirical shortcomings. Epidemiologic Perspectives \& Innovations. http://www.epiperspectives.com/content/1/1/5; Stand: 2004

51 Morfeld P. Schwellenwerte und Dosis-Wirkungs-Beziehungen in epidemiologischen und toxikologischen Studien zu Staubeffekten. In: Herausforderung Feinstaub - Beiträge des Instituts für Arbeitswissenschaften der RAG AG 2006. http://www.econsense.de/_PUBLIKATIONEN/_ECONSENSE_PUBLIK/images/Feinstaubveroeffentlichung.pdf

52 Hurley F, Hunt A, Cowie H et al. Methodology for the Cost-Benefit analysis for CAFE: Volume 2: Health Impact Assessment. AEA Technology Environment/ED51014 2005. http://ec.europa.eu/environment/air/cafe/pdf/cba_methodology_vol2.pdf

53 Roller M. Die Risikoabschätzung des LAI vor dem Hintergrund der Feinstaubdiskussion im Jahre 2005. Gefahrstoffe - Reinhaltung der Luft 2005; 65: $425-434$

54 Wichmann H-E. Positive gesundheitliche Auswirkungen des Einsatzes von Partikelfiltern bei Dieselfahrzeugen - Risikoabschätzung für die Mortalität in Deutschland. Umweltmed Forsch Prax 2004; 9: 85 - 99

55 Schwartz J. Assessing confounding, effect modification, and thresholds in the association between ambient particles and daily deaths. Environ Health Perspect 2000; 108: 563 - 568

56 Stöger T, Reinhard C, Takenaka $S$ et al. Exposure dose-response relationships of instilled carbonaceous nanoparticles indicate a threshold dose for acute inflammation in mice. Environ Health Perspect 2006; 114: $328-333$

57 World Health Organization. Meta-analysis of time-series studies and panel studies of particulate matter (PM) and ozone (O3). Copenhagen, WHO Regional Office for Europe, 2004 (document EUR/04/5042688). http://www.euro.who.int/document/E82792.pdf

58 Elder A, Couderc JP, Gelein R et al. Effects of on-road highway aerosol exposures on autonomic responses in aged, spontaneously hypertensive rats. Inhal Toxicol 2007; 19: 1 - 12

59 Riediker M, Devlin RB, Griggs TR et al. Cardiovascular effects in patrol officers are associated with fine particulate matter from brake wear and engine emissions. Part Fibre Toxicol 2004; 1: 1-10

60 Gilmour PS, Schladweiler MC, Nyska A et al. Systemic imbalance of essential metals and cardiac gene expression in rats following acute pulmonary zinc exposure. J Toxicol Environ Health A 2006; 69: 2011 2032

61 Sandström T, Nowak D, van Bree L. Health effects of coarse particles in ambient air - messages for research and decision-making. Eur Respir J 2005; 26: $187-188$

62 WHO European Centre for environment and health Bonn office. Health risk of particulate matter from long-range transboundary air pollution. DRAFT August 2005. http://www.unece.org/env/wge/200508_Wge24_PmDraft.pdf

63 Brunekreef $B$, Forsberg $B$. Epidemiological evidence of effects of coarse airborne particles on health. Eur Respir J 2005; 26: 309 - 318 